Article

\title{
Neighbor Affinity-Based Core-Attachment Method to Detect Protein Complexes in Dynamic PPI Networks
}

\author{
Xiujuan Lei * and Jing Liang \\ School of Computer Science, Shaanxi Normal University, Xi'an 710119, China; jliang@snnu.edu.cn \\ * Correspondence: xjlei@snnu.edu.cn; Tel.: +86-029-8531-0161
}

Received: 28 June 2017; Accepted: 18 July 2017; Published: 24 July 2017

\begin{abstract}
Protein complexes play significant roles in cellular processes. Identifying protein complexes from protein-protein interaction (PPI) networks is an effective strategy to understand biological processes and cellular functions. A number of methods have recently been proposed to detect protein complexes. However, most of methods predict protein complexes from static PPI networks, and usually overlook the inherent dynamics and topological properties of protein complexes. In this paper, we proposed a novel method, called NABCAM (Neighbor Affinity-Based Core-Attachment Method), to identify protein complexes from dynamic PPI networks. Firstly, the centrality score of every protein is calculated. The proteins with the highest centrality scores are regarded as the seed proteins. Secondly, the seed proteins are expanded to complex cores by calculating the similarity values between the seed proteins and their neighboring proteins. Thirdly, the attachments are appended to their corresponding protein complex cores by comparing the affinity among neighbors inside the core, against that outside the core. Finally, filtering processes are carried out to obtain the final clustering result. The result in the DIP database shows that the NABCAM algorithm can predict protein complexes effectively in comparison with other state-of-the-art methods. Moreover, many protein complexes predicted by our method are biologically significant.
\end{abstract}

Keywords: protein-protein interaction (PPI) network; protein complexes; neighbor affinity; core-attachment

\section{Introduction}

With advances in high-throughput techniques, a lot of protein-protein interaction (PPI) data has been generated [1]. The emergence of large-scale PPI data has raised a hot wave of research on PPI networks in the post-genomic era. Protein interactions are important for most biological process; thus, PPI networks provide a graph of cellular mechanisms. A significant task of system biology is to explore cellular function and organization by analyzing a PPI network [2]. Almost all of the functional processes within a cell are carried out by complexes, which are formed by interaction [3]. Protein complexes participate in specific cellular functions, such as transcription of DNA, translation of mRNA and cell cycle [4]. Protein complexes can help us identify the functions of proteins [5]. The accurate prediction of complexes in PPI networks is significant for understanding the principles of cellular organization and function [6].

So far, many algorithms have been proposed to predict protein complexes from PPI networks. Bader and Hogue [7] proposed the MCODE (molecular complex detection) algorithm. Liu et al. [8] proposed the CMC (clustering based on maximal cliques) algorithm, which predicts complexes based on maximal cliques. MCL (Markov clustering) [9] was applied to identify protein complexes by simulating random walks in PPI networks. Nepusz et al. [10] presented the ClusterONE algorithm to identify protein complexes.

However, these algorithms only focus on the static PPI networks. In fact, PPI networks in cells are dynamic; they change over environment and time [11]. Therefore, the shift from static PPI networks to 
dynamic PPI networks is critical to identify protein complexes accurately. Wang et al. [12] injected gene expression data into static PPI networks to construct dynamic PPI networks and detect complexes. Park and Bader [13] proposed the DHAC (Dynamical Hierarchical Agglomerative Clustering) algorithm to predict temporal protein complexes from dynamic PPI networks. Ou-Yang et al. [14] presented a novel method to predict overlapping temporal protein complexes from dynamic PPI networks. Li et al. [15] presented the DPC algorithm to identify dynamic protein complexes.

In addition, Gavin et al. [16] revealed the inherent property of protein complexes. Protein complexes have a core part and an attachment part. Wu et al. [17] proposed the COACH algorithm, which is based on core attachment. Kouhsar et al. [4] used a semantic similarity measure based on Gene Ontology (GO) structure to give weights between proteins in the PPI networks. Pizzuti and Rombo [18] take advantage of genetic algorithms and six topological-based fitness functions to predict protein complexes.

To identify protein complexes accurately and biologically, researchers should pay attention to the structure properties of protein complexes predicted from dynamic PPI networks. In this paper, we proposed a novel algorithm, called NABCAM (Neighbor Affinity-Based Core-Attachment Method), to identify dynamic protein complexes. First, the centrality score of every protein is calculated. The proteins with the highest centrality scores are regarded as the seed proteins. Second, the seed proteins are expanded to complexes cores by calculating the similarity value between seed proteins and their neighbor proteins. Thirdly, the attachments are appended to their corresponding protein complex cores by comparing the affinity among neighbors inside the cluster against that outside the cluster. Finally, filtering processes are carried out. Therefore, we obtain the protein complexes set from dynamic PPI networks.

The outline of this paper is as follows. Section 2 describes some related theories and the details of our algorithms. Section 3 shows the experimental results and analysis. Section 4 concludes the paper.

\section{Method}

In this section, some relative terminologies that are used in our experiments are presented. Then, we describe the NABCAM algorithm in the following subsections.

\subsection{Dynamic PPI Networks Construction}

The dynamic PPI networks are constructed by integrating the static PPI data and gene expression data [19], because gene expression level and protein expression level are consistent. To identify the timestamps with high expression value of a protein, we use the three-sigma principle [12] to differentiate the active and inactive timestamps of a protein during the cellular cycle. As gene expression data has 12 timestamps, the static PPI network is divided into 12 sub-graphs, which correspond to 12 timestamps. Eventually, the dynamic PPI network is constructed. Figure 1 shows a process of dynamic PPI network construction.

\subsection{Formation Process of Attachment}

In this algorithm, we focus on the inherent organization of protein complexes. Based on the core-attachment structure, our algorithm identified protein complexes in dynamic PPI networks. On the formation process of attachment, we utilize the idea of neighbor affinity. As shown in Figure 2, the proteins inside the black circle constitute a complex core $c$, and the yellow protein is one of $c^{\prime}$ s candidate neighbor proteins to be merged, which is represented by $v$. The neighbors of $v$ inside the core $c$ are in the blue dotted circle, while those outside $c$ are in the green dotted circle. For a protein $v$, its neighbor affinity inside core $c$ and outside core $c$ are defined respectively. If the $N A(v)=N A I(v)-N A O(v)$ is more than the threshold $T n$, the yellow protein will be merged into the core $c$ as the attachment. The rest of the neighbor proteins of core $c$ repeat such a process until no proteins are left to be merged. After the attachment formation, we can obtain the complexes. 


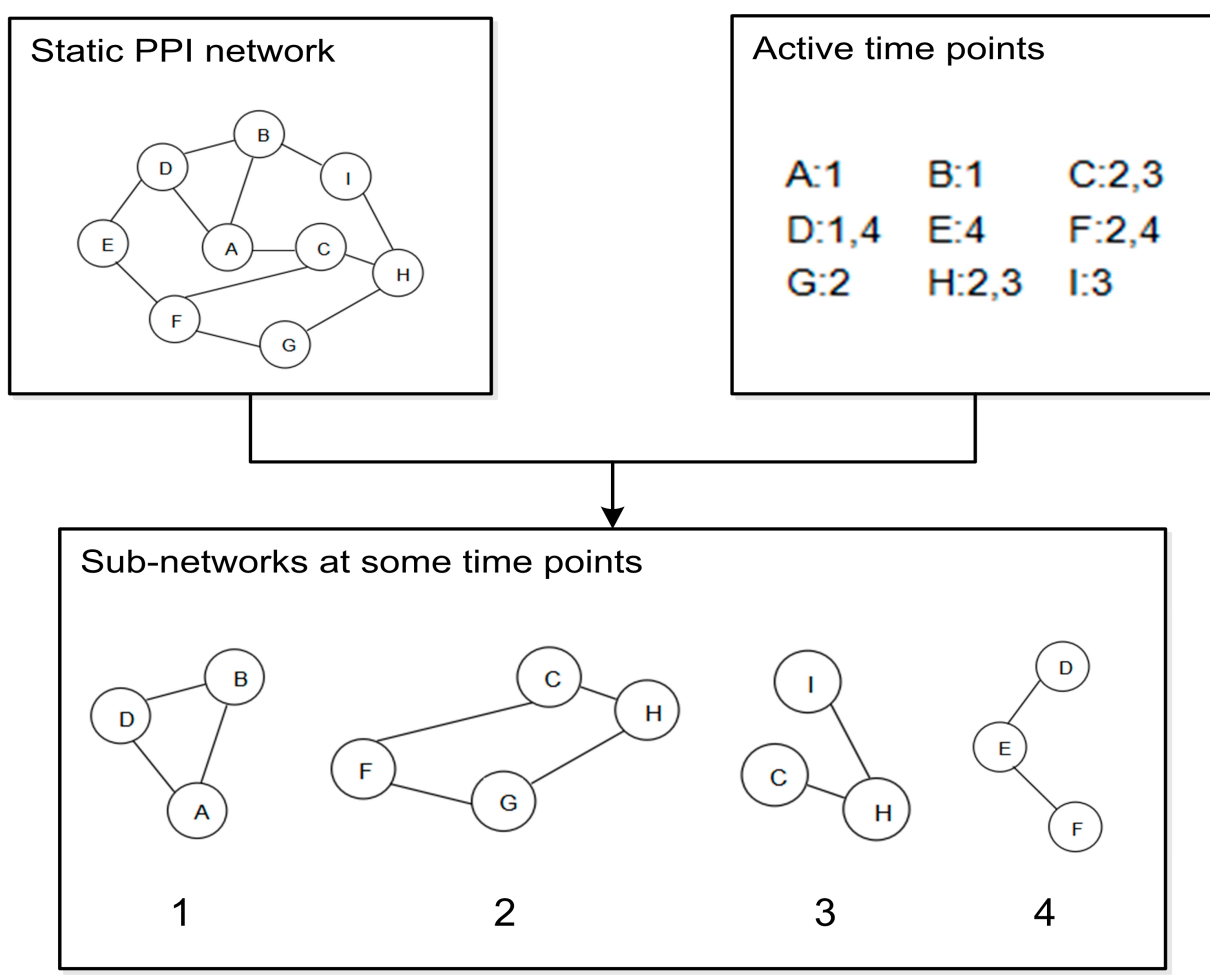

Figure 1. Dynamic protein-protein interaction (PPI) networks construction: (1) the subnet of time point 1; (2) the subnet of time point 2; (3) the subnet of time point 3; (4) the subnet of time point 4.

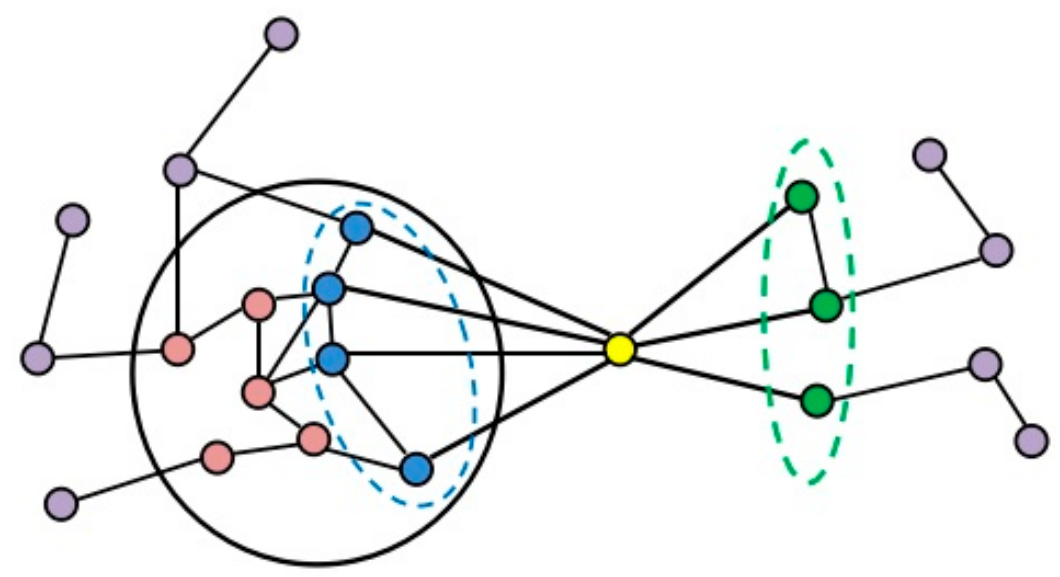

Figure 2. A formation process of attachment: these proteins inside the black circle constitute a complex core; the yellow protein represents a candidate neighbor protein of complex core; the blue proteins represent neighbors inside core; the green proteins represent neighbors outside core.

\subsection{NABCAM Algorithm}

Some analysis of protein complexes revealed the core-attachment structure of a complex [20]. In Figure 3, we visualize a formation process of a protein complex on the PPI networks to clearly describe the NABCAM algorithm. 


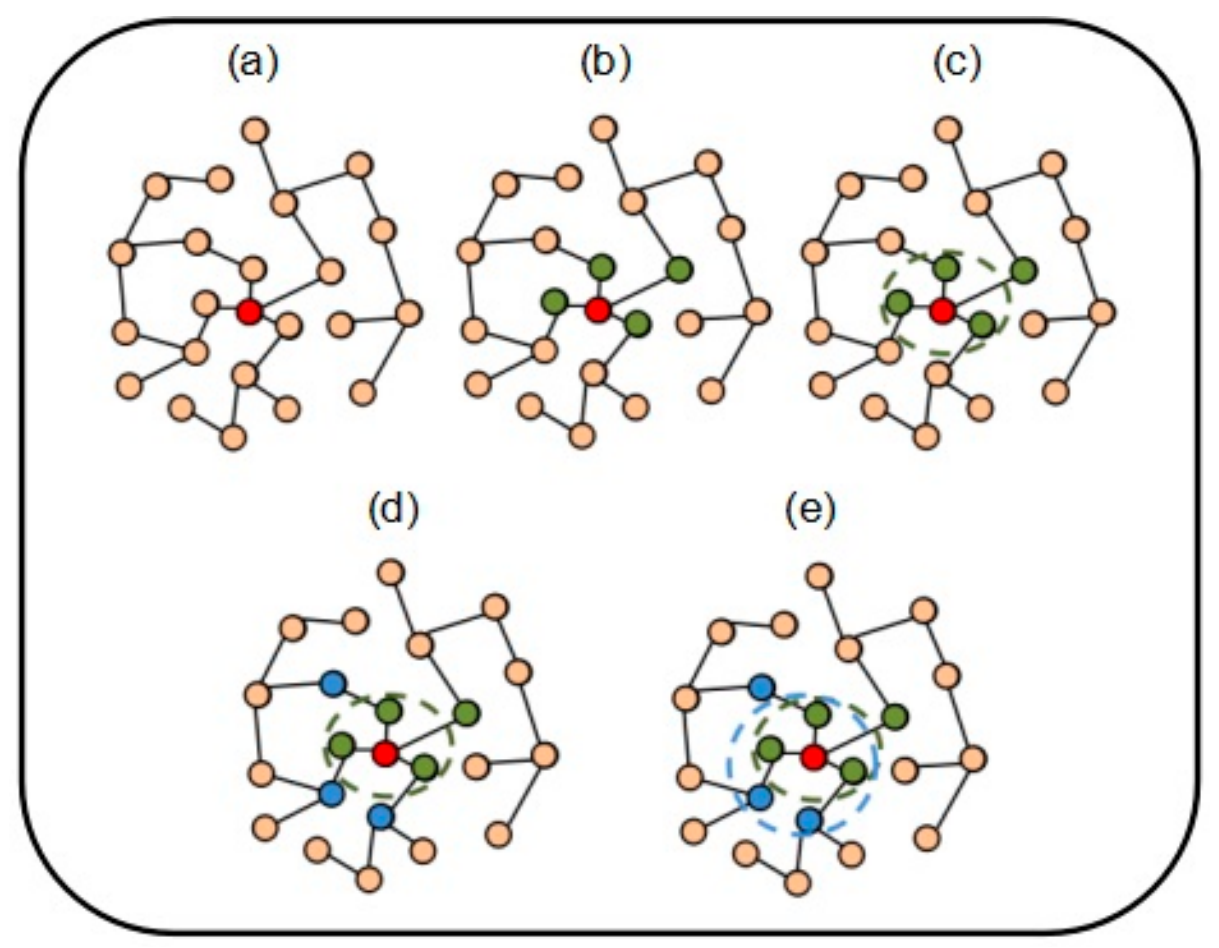

Figure 3. The formation process of a protein complex: (a) the red protein represents the seed protein; (b) the green proteins represent neighbor proteins of the seed protein; (c) these proteins inside the green dotted circle constitute a complex core; (d) the blue proteins represent neighbor proteins of the core; (e) the proteins inside the blue dotted circle constitute a protein complex.

Based on core-attachment structure assumption, the formation process of predicted protein complexes sets involves five phases. The pseudo-code of the NABCAM algorithm is shown in Figure 4 .

In the first phase (Figure 3a), the algorithm selects some seed proteins based on the dense-spread centrality score. For a protein $v \in V$, the $d s c(v)$ is calculated by Equation (1).

$$
\operatorname{dsc}(v)=\operatorname{dens}\left(G_{v}\right) *\left|V_{v}\right|
$$

Considering the density and the size of the induced sub-graph of $v$, the dense-spread centrality score [21] of protein $v$ is defined. For a protein $v$, its induced sub-graph is represented by $G_{v}=\left(V_{v}, E_{v}\right)$, where $V_{v}=\{v \cup N(v)\}$ and $E_{v}=\left\{(u, v) \mid u, v \in V_{v},(u, v) \in E\right\}$. The density of $G_{v}$ is described as following: $\operatorname{dens}\left(G_{v}\right)=2 *\left|E_{v}\right| /\left(\left|V_{v}\right| *\left(\left|V_{v}\right|-1\right)\right)$, where $\left|V_{v}\right|$ represents the number of the proteins involved in $G_{v}$ and $\left|E_{v}\right|$ represents the number of the interactions involved in $G_{v}$. The protein $v$ is added to the seed protein set only if $\operatorname{des}(v)>T s$, where $\operatorname{des}(v)$ is the dense-spread centrality score and $T s$ is the seed threshold. A protein $v$ is discarded if it has a des $(v)$ value less than the threshold value Ts. This is done for all proteins in the PPI networks. The obtained seed proteins are the primary part of the complex cores. 


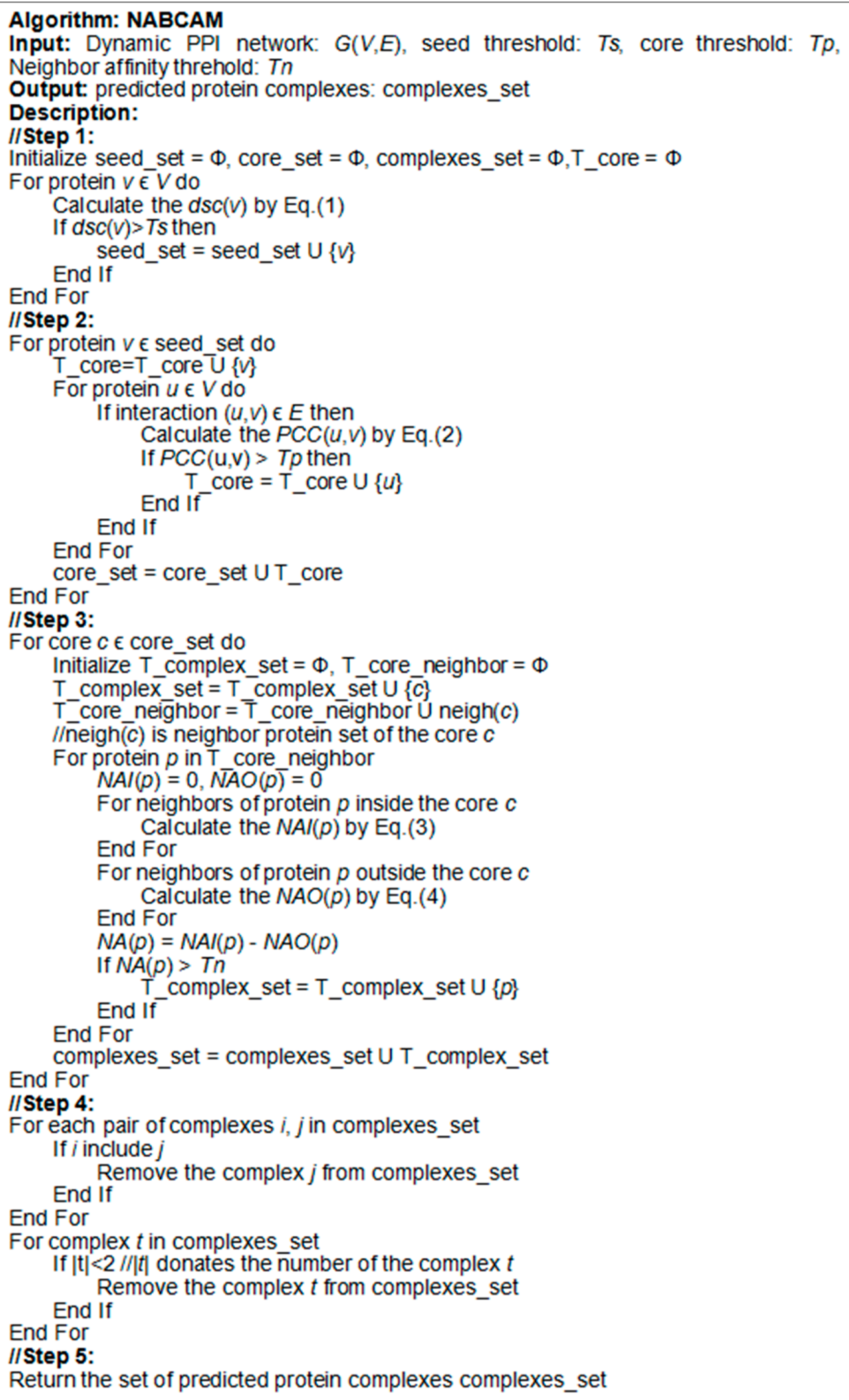

Figure 4. The description of the Neighbor Affinity-Based Core-Attachment Method (NABCAM) algorithm. 
In the second phase (Figure 3b,c), we need to expand the seed proteins to the whole complex core. For a seed protein $v$, we compute the Pearsons correlation coefficient between seed protein $v$ and its neighbor protein $u$. The PCC $(u, v)$ is calculated by Equation (2).

$$
\operatorname{PCC}(X, Y)=\frac{\sum_{i=1}^{n}\left(x_{i}-x^{\prime}\right)\left(y_{i}-y^{\prime}\right)}{\sqrt{\sum_{i=1}^{n}\left(x_{i}-x^{\prime}\right)^{2} \sum_{j=1}^{n}\left(y_{i}-y^{\prime}\right)^{2}}}
$$

where $X=\left\{x_{1}, x_{2}, \ldots, x_{n}\right\}$ and $Y=\left\{y_{1}, y_{2}, \ldots, y_{n}\right\}$ gives the expression values of protein $X$ and $Y$ for $n$ time points, and $x^{\prime}$ and $y^{\prime}$ give the mean of expression values of $X$ and $Y$, respectively. The Pearsons correlation coefficient (PCC) is a measure of the correlation between two proteins $X$ and $Y$ [22]. The more similar the two proteins are, the larger their PCC value. The neighbor protein $u$ is appended to the core whose seed protein is $v$ only if $\operatorname{PCC}(u, v)>T p$, where $\operatorname{PCC}(u, v)$ and Tp is the core threshold. When all of the neighbor proteins of seed proteins are traversed over, we obtain the whole complex cores.

In the third phase (Figure 3d,e), we form a protein complex by selecting the attachments of every complex core's peripheral information. We adopt the neighbor affinity to supplement the attachment for complex cores. For the neighbor protein $p$ of the complex core $c$, we compute separately its affinity among neighbors inside and outside the core $c$, namely the values of $N A I(p)$ and $N A O(p)$. NAI $(p)$ and $N A O(p)$ are calculated by Equations (3) and (4), respectively.

$$
\begin{gathered}
N A I(v)=\frac{\sum_{u \in N I(v)} d(u)}{|N I(v)|} \\
N A O(v)=\frac{\sum_{u \in N O(v)} d(u)}{|N O(v)|}
\end{gathered}
$$

where $v$ is a neighbor protein of $\operatorname{core} c$, and $d(u)$ represents the number of neighbors of protein $u$. NI (v) denotes neighbors of protein $v$ inside the $\operatorname{core} c$, the number of proteins in $N I(v)$ is represented $|N I(v)|$. $N O(v)$ denotes neighbors of protein $v$ outside the core $c$, the number of proteins in $N O(v)$ is represented $|N O(v)|$. The difference between $N A I(p)$ and $N A O(p)$ is denoted by $N A(p)$. If $N A(p)>T n$, the protein $p$ is merged into the core $c$. When all of the neighbor proteins of complex cores are traversed over, we obtain the protein complex set.

In the fourth phase, we should remove redundant protein complexes from the protein complex set. This is a significant step to purify the experimental results. For a protein complex, it may be included in other complexes. In our experiment, for the identified complexes that completely overlap with others, only one can be retained, while the others should be removed as redundant. Moreover, a predicted protein complex may only contain a protein, which also should be removed.

Finally, the NABCAM algorithm is performed on dynamic PPI networks, thereby generating the predicted protein complex set as the result.

Moreover, the seed threshold $T s$, the core threshold Tp and the neighbor affinity threshold Tn, used in the algorithm NABCAM, decide the seed protein's selection, complex cores formation, and protein complexes acquirement, respectively. To find the appropriate thresholds, NABCAM is run with various values of $T s, T p$ and Tn on the DIP, MIPS, and Krogan networks, respectively. In this paper, the appropriate thresholds of $T s, T p$ and $T n$ are $0.3,0.3$ and 0. 


\section{Experiments and Results}

\subsection{Experimental Datasets}

In the present paper, the PPI data of S. cerevisiae from the DIP [23], MIPS [24] and Krogan [25] databases are used to validate the performance of NABCAM algorithm. The dynamic DIP PPI networks are 12 static PPI subnets, corresponding to 12 time points. Different subnets have different scales, as shown in Table 1. It is the same in the MIPS and Krogan datasets. The gold standard dataset of known yeast complexes is derived from CYC2008 [20], which contain 408 complexes and 1628 proteins. The biggest cluster has 81 proteins, while the smallest cluster has two proteins in the complexes of CYC2008.

Table 1. The number of proteins and interactions in each subnet of different PPI networks.

\begin{tabular}{|c|c|c|c|c|c|c|c|c|c|c|c|c|c|}
\hline Data & Timestamp & 1 & 2 & 3 & 4 & 5 & 6 & 7 & 8 & 9 & 10 & 11 & 12 \\
\hline \multirow{2}{*}{ DIP } & Proteins & 797 & 941 & 796 & 623 & 601 & 530 & 493 & 944 & 1090 & 592 & 661 & 461 \\
\hline & Interactions & 981 & 1444 & 1188 & 745 & 750 & 646 & 573 & 1705 & 2185 & 856 & 974 & 526 \\
\hline \multirow{2}{*}{ MIPS } & Proteins & 737 & 897 & 781 & 583 & 570 & 531 & 470 & 839 & 1014 & 523 & 616 & 402 \\
\hline & Interactions & 1097 & 1443 & 1183 & 754 & 684 & 642 & 504 & 1238 & 1637 & 878 & 1207 & 700 \\
\hline \multirow{2}{*}{ Krogan } & Proteins & 336 & 379 & 320 & 256 & 206 & 189 & 202 & 580 & 626 & 304 & 330 & 250 \\
\hline & Interactions & 334 & 464 & 331 & 234 & 210 & 184 & 213 & 1025 & 1081 & 314 & 373 & 258 \\
\hline
\end{tabular}

\subsection{Evaluation Criteria}

To assess the performance of methods, there are three evaluation indicators: precision, recall and $f$-measure [26]. We presented the overlap score (OS) [12] between the predicted protein complexes and gold standard datasets. It can be defined as following Equation (5):

$$
O S(p, g)=\frac{|p \cap g|^{2}}{|p| \cdot|g|}
$$

where $|p|$ is the size of the identified protein complex, $|g|$ is the size of the standard protein complex, and $|p \cap g|$ is the common protein number from the identified and gold complexes. If $O S(p, g) \geq w$, we claim that $p$ and $g$ have been matched. In this paper, we set $w$ to be equal to 0.2 , which is consistent with previous articles [12].

The precision denotes the proportion of the predicted protein complexes perfectly matched by the standard protein complexes in the prediction of the complex. It can be defined by the following Equation (6):

$$
\text { precision }=\frac{N_{c p}}{|P|}
$$

where $|P|$ represents the number of predicted protein complexes, and $N_{c p}$ indicates that the number of the predicted complexes perfectly matched by the known protein complexes. The higher precision is, the more accurate the algorithm is.

The recall indicates the proportion of the known protein complexes perfectly matched by the predicted protein complexes in the standard of the protein complex. It can be defined by the following Equation (7):

$$
\text { recall }=\frac{N_{c b}}{|B|}
$$

where $|B|$ represents the number of known protein complexes, and $N_{c b}$ indicates the number of standard protein complexes perfectly matched by the predicted protein complexes. The higher recall is, the more accurate the algorithm is for predicting protein complexes. 
The precision and recall describe the effectiveness of the algorithm from different aspects. In order to consider these indicators synthetically, the f-measure is defined as the harmonic mean of precision and recall, which can access the overall performance of a method. It is defined by the following Equation (8):

$$
f-\text { measure }=\frac{2 \times \text { precision } \times \text { recall }}{\text { precision }+ \text { recall }}
$$

From the formula of harmonic mean, we can see that precision and f-measure have a relationship of positive correlation. Similarly, recall and f-measure also have a relationship of positive correlation.

In order to further validate the biological significance of protein complexes, we need to carry out the functional enrichment analysis by using the p-value [27] formulated through the following Equation (9):

$$
p-\text { value }=\sum_{i=m}^{n} \frac{\left(\begin{array}{c}
M \\
i
\end{array}\right)\left(\begin{array}{c}
N-M \\
n-i
\end{array}\right)}{\left(\begin{array}{c}
N \\
n
\end{array}\right)}
$$

where $N$ is the number of proteins in the PPI network, $M$ is the number of proteins in a GO term, and $n$ is the number of proteins that are annotated with the same GO term. Generally, the smaller the $p$-value of a protein complex, the stronger the biological significance of the complex processes will be. In this paper, a detected complex is considered to be significant if its $p$-value is less than 0.01 .

\subsection{Comparison with Known Complexes}

In this section, the predicted protein complexes are compared with the standard protein complexes. In Figure 5, we visualize a protein complex to clearly show the performance of the NABCAM algorithm. In Figure 5a, there are 12 proteins in this standard complex. In Figure 5b, there are 12 proteins in the complex we identified. Our algorithm predicted 11 proteins accurately. The protein YHR081W is the missed protein, and the protein YMR128W is detected falsely.

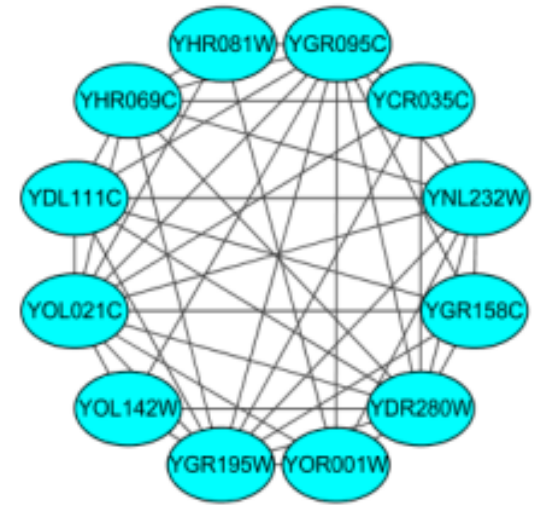

(a)

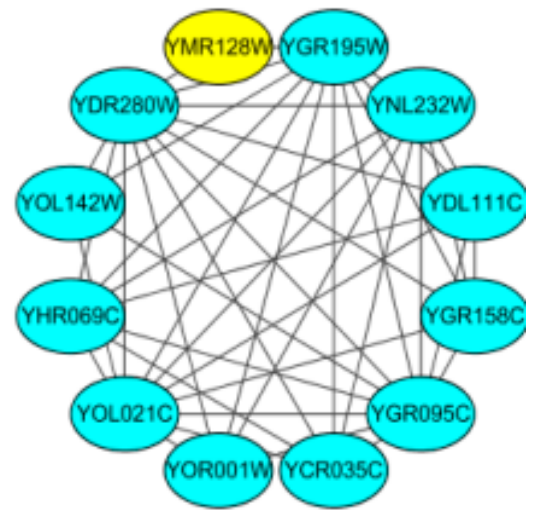

(b)

Figure 5. Visualization of a protein complex: (a) standard complex; (b) identified complex: the yellow protein represents the wrong protein; the blue proteins represent correct proteins.

\subsection{Comparison Based on Precision, Recall and F-Measure}

As shown in Figure 6, we compared our algorithm on dynamic DIP PPI networks with the following state-of-the-art protein complex prediction algorithms: MOEPGA [28], HC-PIN [29], MCL [30], DPClus [31], RNSC [23], COACH [17], CORE [32], ClusterOne [10], CFinder [33], MCODE [7], and CMC [8]. When using the dynamic DIP PPI networks, the NABCAM method 
achieves precision, recall and $f$-measure values of $0.6903,0.4917$ and 0.5743 , respectively. It is obvious that the precision value of our method is much more excellent than other prediction methods. Compared with other methods, our algorithm's recall value is a little lower than the recall values of MOEPGA, DPClus, $\mathrm{COACH}$ and CMC. However, the $f$-measure is higher for the NABCAM algorithm than its counterpart methods. The other methods MOEPGA, HC-PIN, MCL, DPClus, RNSC, COACH, CORE, ClusterOne, CFinder, MCODE and CMC achieved $f$-measure values of $0.4510,0.3600,0.3717,0.4653$, $0.4359,0.5019,0.4766,0.3680,0.4331,0.3342$ and 0.4100 .

Moreover, we also compare our method with the following established leading protein complex prediction methods: CSO [34], ClusterOne [10], COACH [17], CMC [8], HUNTER [35], and MCODE [7] in terms of precision, recall and f-measure in the MIPS and Krogran datasets, respectively, as shown in Figures 7 and 8. As shown in Figure 7, our method achieves the highest $f$-measure of 0.5382 , recall of 0.5094, and precision of 0.5706 in MIPS dataset, which obviously outperforms other methods. In Figure 8, it can be seen that our method achieves the highest $f$-measure of 0.5575 , recall of 0.4259 and precision of 0.8068 in the Krogan dataset, which obviously outperforms other methods.

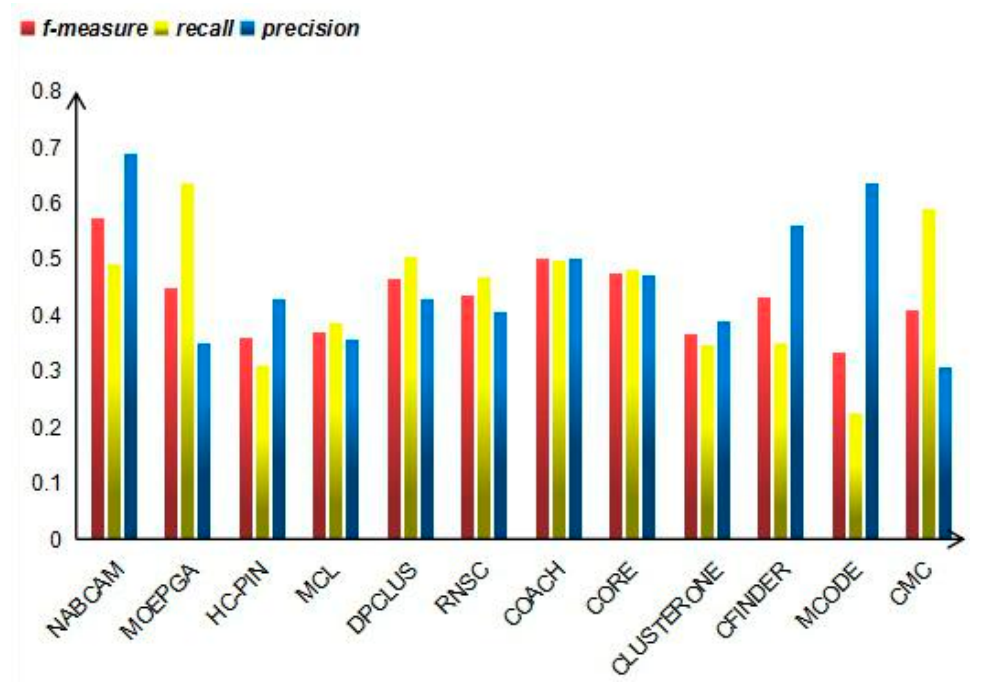

Figure 6. Precision, recall and f-measure values of various algorithms on the DIP dataset.

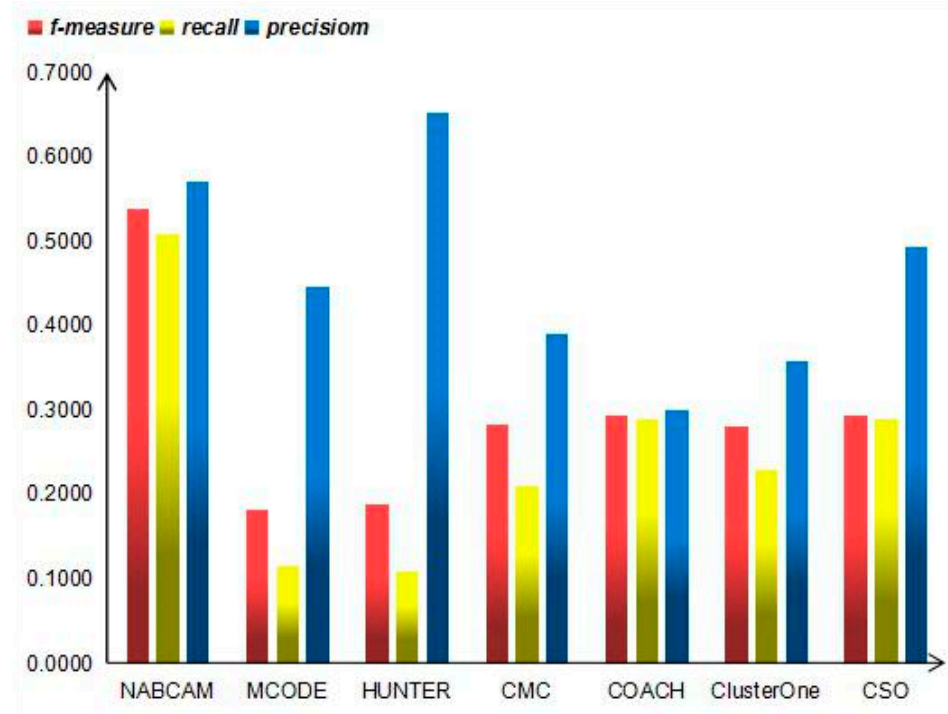

Figure 7. Precision, recall and f-measure values of various algorithms on the MIPS dataset. 


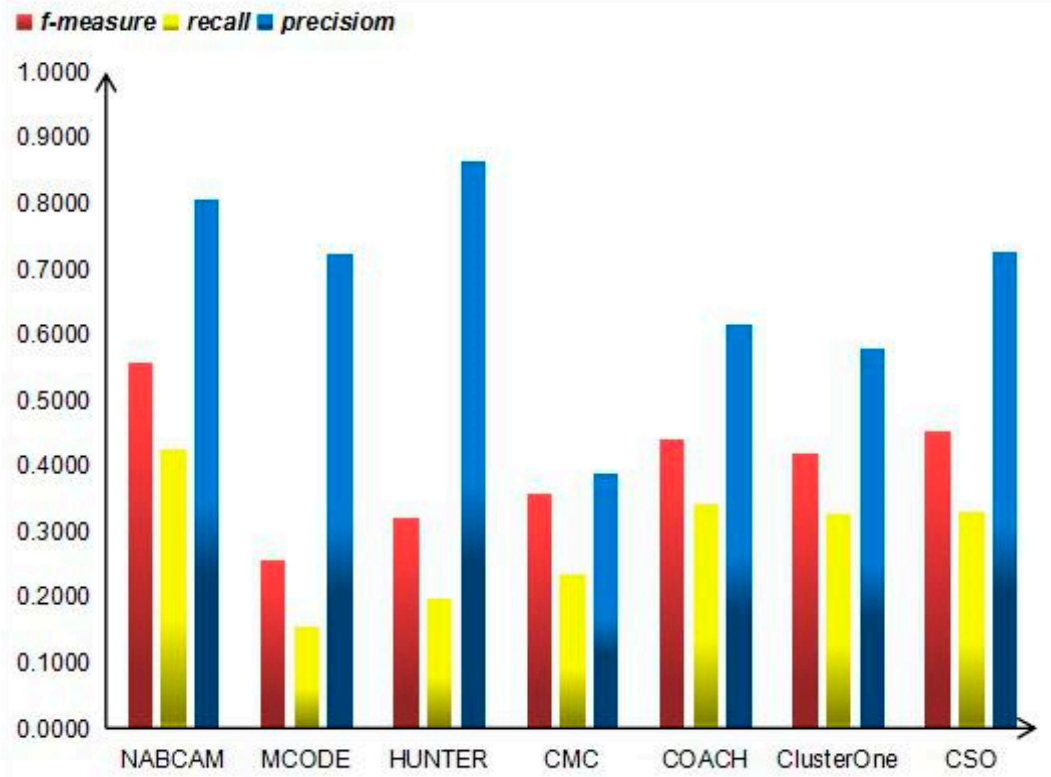

Figure 8. Precision, recall and f-measure values of various algorithms on the Krogan dataset.

\subsection{Comparison Based on Gene Ontology (GO) Semantic}

A complex is considered significant when its $p$-value is less than 0.01 . In this experiment, we use the tool GO::TermFinder [21] to calculate the $p$-value of identified complexes whose size is greater than two.

Table 2 lists the number and percentage of the predicted protein complexes whose $p$-value is in the range of $<10^{-15},\left[10^{-15}, 10^{-10}\right),\left[10^{-10}, 10^{-5}\right),\left[10^{-5}, 0.01\right), \geq 0.01$. Table 2 shows the comparison of the functional enrichment of complexes identified by NABCAM, MCL, CORE and ClusterOne, in the DIP, MIPS and Krogan datasets. As shown in Table 2, we can obtain the number of predicted protein complexes by different methods on different datasets. The percentage and the amount of the predicted protein complexes with $p$-values greater than 0.01 fall into corresponding intervals. We can see from Table 2 that our algorithm outperforms the MCL, CORE and ClusterOne algorithms. In the DIP dataset, the percentage of complexes whose $p$-value is greater than 0.01 in predicted complexes by the NABCAM algorithm is the smallest. So, most of the predicted protein complexes by the NABCAM algorithm are significant. Similarly, we can obtain results on the MIPS and Krogan datasets. The results illustrate that the NABCAM algorithm is competent at identifying significant protein complexes in PPI networks.

Table 2. Functional enrichment analysis of complexes detected on different datasets.

\begin{tabular}{cccccccc}
\hline Data & Algorithm & PC & $<\mathbf{1 0}^{-\mathbf{1 5}}$ & {$\left[\mathbf{1 0}^{-\mathbf{1 5}}, \mathbf{1 0 ^ { - 1 0 }}\right)$} & {$\left[\mathbf{1 0}^{-\mathbf{1 0}}, \mathbf{1 0}^{-\mathbf{5}}\right)$} & {$\left[\mathbf{1 0}^{-\mathbf{5}}, \mathbf{0 . 0 1}\right)$} & $\geq \mathbf{0 . 0 1}$ \\
\hline \multirow{5}{*}{ DIP } & NABCAM & 1702 & $136(7.99 \%)$ & $230(13.51 \%)$ & $820(48.18 \%)$ & $343(20.15 \%)$ & $173(10.16 \%)$ \\
& MCL & 1053 & $19(1.80 \%)$ & $47(4.46 \%)$ & $183(17.38 \%)$ & $362(34.38 \%)$ & $442(41.98 \%)$ \\
& CORE & 344 & $1(0.29 \%)$ & $3(0.87 \%)$ & $78(22.67 \%)$ & $114(33.14 \%)$ & $148(43.02 \%)$ \\
& ClusterOne & 574 & $21(3.66 \%)$ & $52(9.06 \%)$ & $177(30.84 \%)$ & $184(32.06 \%)$ & $140(24.39 \%)$ \\
\hline \multirow{5}{*}{ MIPS } & NABCAM & 966 & $30(3.10 \%)$ & $70(7.25 \%)$ & $332(34.37 \%)$ & $333(34.47 \%)$ & $201(20.81 \%)$ \\
& MCL & 606 & $5(0.83 \%)$ & $13(2.15 \%)$ & $94(15.51 \%)$ & $220(36.30 \%)$ & $274(45.21 \%)$ \\
& CORE & 340 & $0(0.00 \%)$ & $4(1.18 \%)$ & $65(19.12 \%)$ & $107(31.47 \%)$ & $164(48.24 \%)$ \\
& ClusterOne & 372 & $7(1.88 \%)$ & $16(4.30 \%)$ & $117(31.45 \%)$ & $126(33.87 \%)$ & $106(28.49 \%)$ \\
\hline \multirow{5}{*}{ Krogan } & NABCAM & 587 & $75(12.78 \%)$ & $75(12.78 \%)$ & $304(51.79 \%)$ & $108(18.39 \%)$ & $25(4.26 \%)$ \\
& MCL & 403 & $16(3.97 \%)$ & $43(10.67 \%)$ & $103(25.56 \%)$ & $119(29.53 \%)$ & $122(30.27 \%)$ \\
& CORE & 255 & $3(1.18 \%)$ & $10(3.92 \%)$ & $60(23.53 \%)$ & $102(40.00 \%)$ & $80(31.37 \%)$ \\
& ClusterOne & 399 & $13(3.26 \%)$ & $43(10.78 \%)$ & $98(24.56 \%)$ & $120(30.08 \%)$ & $125(31.33 \%)$ \\
\hline
\end{tabular}


To further reveal the biological significance of predicted complexes, five identified protein complexes with different datasets are presented in Table 3, which lists the $p$-value of protein complexes, cluster frequency, and the Gene Ontology term.

Table 3. Predicted protein complexes with small p-values on different datasets.

\begin{tabular}{|c|c|c|c|c|}
\hline Data & ID & $p$-Value & Cluster Frequency & Gene Ontology Term \\
\hline \multirow{5}{*}{ DIP } & 1 & $2.45 \times 10^{-47}$ & 30 out of 34 genes, $88.2 \%$ & ribosomal small subunit biogenesis \\
\hline & 2 & $4.48 \times 10^{-38}$ & 22 out of 23 genes, $95.7 \%$ & mRNA splicing, via spliceosome \\
\hline & 3 & $1.41 \times 10^{-37}$ & 21 out of 21 genes, $100.0 \%$ & mRNA splicing, via spliceosome \\
\hline & 4 & $3.88 \times 10^{-36}$ & 22 out of 23 genes, $95.7 \%$ & ribosomal small subunit biogenesis \\
\hline & 5 & $1.45 \times 10^{-33}$ & 12 out of 12 genes, $100.0 \%$ & polyadenylation-dependent snoRNA $3^{\prime}$-end processing \\
\hline \multirow{5}{*}{ MIPS } & 1 & $9.62 \times 10^{-27}$ & 16 out of 18 genes, $88.9 \%$ & ribosomal large subunit biogenesis \\
\hline & 2 & $1.15 \times 10^{-25}$ & 18 out of 25 genes, $72.0 \%$ & mitotic sister chromatid segregation \\
\hline & 3 & $1.15 \times 10^{-23}$ & 16 out of 17 genes, $94.1 \%$ & mitotic nuclear division \\
\hline & 4 & $4.02 \times 10^{-23}$ & 14 out of 16 genes, $87.5 \%$ & ribosomal large subunit biogenesis \\
\hline & 5 & $2.69 \times 10^{-22}$ & 16 out of 23 genes, $69.6 \%$ & mitotic sister chromatid segregation \\
\hline \multirow{5}{*}{ Krogan } & 1 & $1.36 \times 10^{-34}$ & 17 out of 18 genes, $94.4 \%$ & ncRNA transcription \\
\hline & 2 & $3.69 \times 10^{-34}$ & 13 out of 15 genes, $86.7 \%$ & tRNA catabolic process \\
\hline & 3 & $2.49 \times 10^{-33}$ & 13 out of 14 genes, $92.9 \%$ & chromatin disassembly \\
\hline & 4 & $3.17 \times 10^{-32}$ & 13 out of 16 genes, $81.2 \%$ & $\begin{array}{l}\text { exonucleolytic trimming to generate mature } \\
3^{\prime} \text {-end of } 5.8 \mathrm{~S} \text { rRNA from tricistronic rRNA transcript } \\
\text { (SSU-rRNA, 5.8S rRNA, LSU-rRNA) }\end{array}$ \\
\hline & 5 & $5.79 \times 10^{-32}$ & 18 out of 18 genes, $100.0 \%$ & mRNA splicing, via spliceosome \\
\hline
\end{tabular}

\section{Conclusions}

In the post-genomic era, it's significant to understand the topological organization of PPI networks, predict protein complexes and discover the functions of proteins. For the sake of these goals, a number of prediction algorithms have been proposed. In this paper, we proposed a novel algorithm, NABCAM, for the computational prediction of protein complexes on dynamic PPI networks. In the NABCAM method, first, some proteins with high dense-spread centrality scores are regarded as seed proteins. Second, the seed proteins are expanded to complexes cores by calculating the similarity value between the seed protein and its neighbor protein. And then the attachments are appended to their corresponding protein complex cores by comparing the affinity among neighbors inside the cluster against that outside the cluster. Our method considers the dynamic properties of PPI networks and the inherent organization of complexes.

Our algorithm is evaluated and analyzed by comparing it with other state-of-the-art algorithms in terms of precision, recall and f-measure. Experimental results show that the NABCAM algorithm has a better performance than other methods. Moreover, a number of protein complexes with strong biological significance are identified from dynamic PPI networks by our algorithm. In the future, we will attempt to apply our algorithm to other organisms.

Acknowledgments: This paper is supported by the National Natural Science Foundation of China (61672334, 61502290, 61401263), Industrial Research Project of Science and Technology in Shaanxi Province (2015GY016).

Author Contributions: X.L. conceptualized the algorithm and designed the method, X.L. and J.L. drafted the manuscript, J.L. performed the experiments and analyzed the data and X.L. modified the manuscript and polished the English expression. All the authors read and approved the manuscript.

Conflicts of Interest: The authors have declared that no competing interests exist.

\section{References}

1. Wang, Y.; You, Z.; Li, X.; Chen, X.; Jiang, T.; Zhang, J. PCVMZM: Using the probabilistic classification vector machines model combined with a zernike moments descriptor to predict protein-protein interactions from protein sequences. Int. J. Mol. Sci. 2017, 18, 1029. [CrossRef] [PubMed] 
2. Zeng, X.; Zhang, X.; Zou, Q. Integrative approaches for predicting microRNA function and prioritizing disease-related microRNA using biological interaction networks. Brief. Bioinform. 2016, 17, 193-203. [CrossRef] [PubMed]

3. Almedia, R.M.; Acqua, S.D.; Krippahl, L.; Moura, J.J.G.; Pauleta, S.R. Predicting protein-protein interactions using bigger: Case studies. Molecules 2016, 21, 1037. [CrossRef] [PubMed]

4. Georgiou, D.N.; Karakasidis, T.E.; Megaritis, A.C. A short survey on genetic sequences, chou's pseudo amino acid composition and its combination with fuzzy set theory. Open Bioinform. J. 2013, 7, 41-48. [CrossRef]

5. Ohno, M.; Karagiannis, P.; Taniguchi, Y. Protein expression analyses at the single cell level. Molecules 2014, 19, 13932-13947. [CrossRef] [PubMed]

6. Ding, Y.; Tang, J.; Guo, F. Identification of protein-protein interactions via a novel matrix-based sequence representation model with amino acid contact information. Int. J. Mol. Sci. 2016, 17, 1623. [CrossRef] [PubMed]

7. Bader, G.D.; Hogue, C.W.V. An automated method for finding molecular complexes in large protein interaction networks. BMC Bioinform. 2003, 4, 2. [CrossRef]

8. Liu, G.; Wong, L.; Chua, H.N. Complex discovery from weighted PPI networks. Bioinform. 2009, 25, 1891-1897. [CrossRef] [PubMed]

9. Srihari, S.; Ning, K.; Leong, H.W. MCL-CAw: A refinement of MCL for detecting yeast complexes from weighted PPI networks by incorporating core-attachment structure. BMC Bioinform. 2010, 11, 504. [CrossRef] [PubMed]

10. Nepusz, T.; Yu, H.; Paccanaro, A. Detecting overlapping protein complexes in protein-protein interaction networks. Nat. Methods 2012, 9, 471-472. [CrossRef] [PubMed]

11. Chen, B.; Fan, W.; Liu, J.; Wu, F.X. Identifying protein complexes and functional modules-from static PPI networks to dynamic PPI networks. Brief. Bioinform. 2014, 15, 177-194. [CrossRef] [PubMed]

12. Wang, J.; Peng, X.; Li, M.; Pan, Y. Construction and application of dynamic protein interaction network based on time course gene expression data. Proteomics 2013, 13, 301-312. [CrossRef] [PubMed]

13. Park, Y.; Bader, J. How networks change with time. Bioinformatics 2012, 28, 40-48. [CrossRef] [PubMed]

14. Ou-Yang, L.; Dai, D.Q.; Li, X.L.; Wu, M.; Zhang, X.F.; Yang, P. Detecting temporal protein complexes from dynamic protein-protein interaction networks. BMC Bioinform. 2014, 15, 335-348. [CrossRef] [PubMed]

15. Li, M.; Chen, W.; Wang, J.; Wu, F.X.; Pan, Y. Identifying dynamic protein complexes based on gene expression profiles and PPI networks. BioMed Res. Int. 2014. [CrossRef] [PubMed]

16. Gavin, A.C.; Aloy, P.; Grandi, P.; Krause, R.; Boesche, M.; Marzioch, M.; Rau, C.; Jensen, L.J.; Bastuck, S.; Dümpelfeld, B. Proteome survey reveals modularity of the yeast cell machinery. Nature 2006, 440, 631-636. [CrossRef] [PubMed]

17. Wu, M.; Li, X.; Kwoh, C.K.; Ng, S.K. A core-attachment based method to detect protein complexes in PPI networks. BMC Bioinform. 2009, 10, 169-184. [CrossRef] [PubMed]

18. Pizzuti, C.; Rombo, S. Experimental evaluation of topological-based fitness functions to detect complexes in PPI networks. In Proceedings of the 14th annual conference on Genetic and evolutionary computation, Philadelphia, PA, USA, 7-11 July 2012; pp. 193-200.

19. Pan, J.; Hu, S.; Wang, H.; Zou, Q. Pagefinder: Quantitative identification of spatiotemporal pattern genes. Bioinformatics 2012, 28, 1544-1545. [CrossRef] [PubMed]

20. Dezso, Z.; Oltvai, Z.N.; Barabasi, A.L. Bioinformatics analysis of experimentally determined protein complexes in the yeast Saccharomyces cerevisiae. Genome Res. 2003, 13, 2450-2454. [CrossRef] [PubMed]

21. Peng, X.; Wang, J.; Huan, J.; Wu, F.X. Double-layer clustering method to predict protein complexes based on power-law distribution and protein sublocalization. J. Theor. Biol. 2016, 395, 186-193. [CrossRef] [PubMed]

22. Jiang, D.; Tang, C.; Zhang, A. Cluster analysis for gene expression data: A survey. IEEE Trans. Knowl. Data Eng. 2004, 16, 1370-1386. [CrossRef]

23. King, A.D.; Pržulj, N.; Jurisica, I. Protein complex prediction via cost-based clustering. Bioinformatics 2004, 20, 3013-3020. [CrossRef] [PubMed]

24. Güldener, U.; Münsterkötter, M.; Oesterheld, M.; Pagel, P.; Ruepp, A.; Mewes, H. Mpact: The MIPS protein interaction resource on yeast. Nucleic Acids Res. 2006, 34, 436-441. [CrossRef] [PubMed]

25. Krogan, N.; Cagney, G.; Yu, H.; Zhong, G.; Guo, X.; Ignatchenko, A. Global landscape of protein complexes in the yeast Saccharomyces cerevisiae. Nature 2006, 440, 637-643. [CrossRef] [PubMed] 
26. Keretsu, S.; Sarmah, R. Weighted edge based clustering to identify protein complexes in protein-protein interaction networks incorporating gene expression profile. Comput. Biol. Chem. 2016, 65, 69-79. [CrossRef] [PubMed]

27. Shen, X.; Yi, L.; Jiang, X.; Zhao, Y.; Hu, X.; He, T.; Yang, J. Neighbor affinity based algorithm for discovering temporal protein complex from dynamic PPI network. Methods 2016, 110, 90-96. [CrossRef] [PubMed]

28. Cao, B.; Luo, J.; Liang, C.; Wang, S.; Song, D. Moepga: A novel method to detect protein complexes in yeast protein-protein interaction networks based on multiobjective evolutionary programming genetic algorithm. Comput. Biol. Chem. 2015, 58, 173-181. [CrossRef] [PubMed]

29. Wang, J.; Li, M.; Chen, J.; Pan, Y. A fast hierarchical clustering algorithm for functional modules discovery in protein interaction networks. Comput. Biol. Bioinform. 2011, 8, 607-620.

30. Leal, J.P.; Enright, A.; Ouzounis, C.A. Detection of functional modules from protein interaction networks. Proteins Struct. Funct. Bioinform. 2003, 54, 49-57. [CrossRef] [PubMed]

31. Altaf-UI-Amin, M.; Shinbo, Y.; Mihara, K.; Kurokawa, K.; Kanaya, S. Development and implementation of an algorithm for detection of protein complexes in large interaction networks. BMC Bioinform. 2006, 7, 207-219. [CrossRef] [PubMed]

32. Leung, H.C.; Xiang, Q.; Yiu, S.M.; Chin, F.Y. Predicting protein complexes from PPI data: A core-attachment approach. J. Comput. Biol. 2009, 16, 133-144. [CrossRef] [PubMed]

33. Adamcsek, B.; Palla, G.; Farkas, I.J.; Vicsek, T. CFinder: Locating cliques and overlapping modules in biological networks. Bioinformatics 2006, 22, 1021-1023. [CrossRef] [PubMed]

34. Zhang, Y.; Lin, H.; Yang, Z.; Wang, J.; Li, Y.; Xu, B. Protein complex prediction in large ontology attributed protein-protein interaction networks. IEEE/ACM Trans. Comput. Biol. Bioinform. 2013, 10, 729-741. [CrossRef] [PubMed]

35. Chin, C.; Chen, S.; Ho, C.; Ko, M.; Lin, C. A hub-attachment based method to detect functional modules from confidence-scored protein interactions and expression profiles. BMC Bioinform. 2010, 11, S25. [CrossRef] [PubMed]

Sample Availability: Samples of the compounds are available from the authors.

(c) 2017 by the authors. Licensee MDPI, Basel, Switzerland. This article is an open access article distributed under the terms and conditions of the Creative Commons Attribution (CC BY) license (http:/ / creativecommons.org/licenses/by/4.0/). 